\title{
Spatiotemporal Time Series Analysis Methods for the Study of Turbulent Magnetohydrodynamic Channel Flows
}

\author{
A. D. Fragkou ${ }^{1}$ - T. E. Karakasidis ${ }^{1}$ - I. E. Sarris ${ }^{2}$ • \\ A. Liakopoulos ${ }^{1}$
}

Received: 12 February 2015 / Accepted: 22 July 2015 / Published online: 31 July 2015

(C) Springer International Publishing Switzerland 2015

\begin{abstract}
In the present study, the direct numerical simulation of the turbulent flow of an electrically conductive fluid in a channel is performed and time series are recorded at a range of locations along the y-direction between the parallel plates of the channel. An external streamwise magnetic field is applied and the correlations between pairs of time series of the fluctuating velocities from the magnetohydrodynamic and the hydrodynamic cases measured at the same probing location of the channel are analyzed. We compare the situation with and without magnetic field using the nonlinear method of Cross Recurrence Plots and Cross Recurrence Quantification Analysis (CRQA). The results of CRQA indicate very well the spatiotemporal extension of the effect of the applied magnetic field on the flow and are in very good agreement with the detailed analysis obtained with other methods.
\end{abstract}

Keywords Cross recurrence plots · Cross recurrence quantification analysis - Turbulent channel flow . Direct Numerical Simulation (DNS)

\section{Introduction}

Only a few direct numerical simulations (DNS) or large-eddy simulations (LES) have been reported for the study of magnetohydrodynamic (MHD) turbulent channel flow with electrically isolated walls. Since for laboratory and industrial scale flows of conducting fluids (except plasmas) the magnetic Reynolds number, $R_{m}$, is much less than unity, the induced magnetic

\section{A. D. Fragkou}

fthanos@uth.gr

1 Laboratory of Hydromechanics and Environmental Engineering, Department of Civil Engineering, University of Thessaly, 38334 Volos, Greece

2 Department of Energy Technology, Technological Educational Institute of Athens, 12210 Athens, Greece 
field is very small in comparison with the externally applied magnetic field and the low- $R_{m}$ approximation is valid for the study of this class of turbulent flows (Moreau 1998). Lee and Choi (2001) have reported a DNS study with the magnetic field to be oriented in $\mathrm{x}, \mathrm{y}$, and zdirections, i.e., the streamwise, the wall-normal and the spanwise directions, respectively. Satake et al. (2006) and Boeck et al. (2007) have reported DNS studies of the turbulent MHD channel flow for high Reynolds numbers, $R e$, considering the wall-normal oriented external magnetic field. The MHD channel flow with an external magnetic field normal to the walls, is usually referred to as the Hartmann flow. Hartmann (1937) performed the first theoretical investigation of magnetohydrodynamic flows, and also, Hartmann and Lazarus (1937) conducted the first experimental research on the influence of a wall-normal magnetic field on turbulent flows. The effect of a magnetic field on turbulence, naturally, results in a change in turbulent transfer of momentum. In the case of turbulent channel flow of liquid metals a wallnormal magnetic field interacts with both the averaged flow and the turbulent fluctuations, while a streamwise (as in the present study) or a spanwise magnetic field interacts only with the turbulent fluctuations.

In the present study, velocities are recorded at the same locations of the channel in the presence of magnetic field and without magnetic field. The time series located at the same channel positions with and without magnetic field are studied using phase space reconstruction methods and more specifically the Cross Recurrence Plots (CRPs) method (Eckmann et al. 1987; Marwan 2003) and the Cross Recurrence Quantification Analysis (CRQA) (Webber and Zbilut 1994; Marwan et al. 2003, 2007) which extracts quantitative information from CRPs. CRQA is the extension of the Recurrence Quantification Analysis method which has been used in the study of various dynamical systems such as proteins (Zbilut et al. 2004; Giuliani et al. 2002), corrosion (Cazares - Ibanez et al. 2005), financial systems (Strozzi et al. 2002; Fabretti and Ausloos 2005), physiological systems (Marwan et al. 2002a, b; Marwan and Meinke 2004; Riley et al. 1999), molecular systems (Karakasidis et al. 2007), among many others. The CRP method is applied on climatological and geophysical data (Marwan et al. 2002a, b; 2003), financial data (Addoa et al. 2013; Crowley and Aaron 2010), engineering data (Nichols et al. 2006; Shockley et al. 2002; Serrà et al. 2009; Wang et al. 2012), astronomical data (Deng et al. 2013; Ponyavin and Zolotova 2004; Sparavigna 2008), Physics and Mathematics (Ganapathy et al. 2007), Biology (Li and Li 2013) etc.

In the present paper, the Cross Recurrence Plots method is employed in order to examine if one can extract qualitative information about the extent of the effect of the magnetic field on the spatial behavior of the flow. The paper is organized as follows. In section 2, the physical problem and the simulation details are given along with the recorded quantities. Section 3 contains a description of the Cross Recurrence Plots and Cross recurrence Quantification Analysis. In section 4, we present the results of our analysis as well a relative discussion of the results. Finally, section 5 presents the conclusions of the present work.

\section{Problem Setup and Governing Equations}

\subsection{Low Magnetic Reynolds Number Limit}

In the limit of low magnetic Reynolds number $\left(R_{m}<<1\right)$ the induced magnetic field is very small when compared to the externally imposed magnetic field $\mathbf{B}_{\mathbf{0}}$ and the electric current $\mathbf{J}$ is given by the Ohm's law (Moreau 1998): 


$$
\mathbf{J}=\sigma\left(-\nabla \Phi+\mathbf{v} \times \mathbf{B}_{\mathbf{0}}\right)
$$

where, $\sigma$ is the electrical conductivity, $\Phi$ the electric potential, (given by $E=-\nabla \Phi$ where $E$ is the electric field) and $\mathbf{v}$ is the velocity field. A discussion about the limits of validity of the low$R_{m}$ approximation for thermal convection flows can be found in Sarris et al. (2006).

It is traditional to consider the governing equations for the velocity in terms of nondimensional variables (Sarris et al. 2007), obtained by introducing a characteristic velocity scale $U$ and a characteristic magnetic field $B$, and as usual for channel flows, Fig. 1, the characteristic scales are chosen to be the channel half-width $\delta$. Introducing also the nondimensional quantities: $\mathbf{b}_{\mathbf{0}}=\mathbf{B}_{\mathbf{0}} / B, \mathbf{u}=\mathbf{v} / U, \mathbf{j}=\mathbf{J} /(\sigma U B)$ and $\phi=\Phi \delta /(U B)$, the following equations for an electrically conducting incompressible fluid in the limit of $R_{m}<<1$ are obtained:

$$
\begin{gathered}
\frac{\partial \mathbf{u}}{\partial t}+(\mathbf{u} \cdot \nabla) \mathbf{u}=-\nabla p+\frac{1}{R e} \nabla^{2} \mathbf{u}+N\left(\mathbf{j} \times \mathbf{b}_{\mathbf{0}}\right) \\
\mathbf{j}=-\nabla \phi+\mathbf{u} \times \mathbf{b}_{\mathbf{0}} \\
\nabla^{2} \phi=\nabla\left(\mathbf{u} \times \mathbf{b}_{\mathbf{0}}\right)=\mathbf{b}_{\mathbf{0}} \cdot \omega
\end{gathered}
$$

where Eq. (4) is a consequence of $\nabla \cdot \mathbf{j}=0$ and is obtained by taking the divergence of Eq. (3). Here $\omega=\nabla \times \mathbf{u}, p$ is the dimensionless pressure and the incompressibility imposes $\nabla \cdot \mathbf{u}=0$. The hydrodynamic Reynolds number is defined as $R e=U \delta / v$.

The traditional choice $B=\left|\mathbf{B}_{\mathbf{0}}\right|$ has been adopted for the characteristic magnetic field so that $\mathbf{b}_{\mathbf{0}}$ is a unit vector in the direction of the magnetic field. For the magnetohydrodynamic case considered here, $\mathbf{b}_{\mathbf{0}}$ can only be aligned in the streamwise direction of the channel. The Stuart number (or interaction parameter), $N=\sigma B^{2} \delta / \rho U$, is the ratio of the electromagnetic force to the inertial force and it is related to the Hartmann number by $H a=\sqrt{\operatorname{Re} N}(=B \delta \sqrt{\sigma / \rho v})$ where $\rho$ is the density of the fluid and $v$ is the kinematic viscosity. The characteristic velocity is chosen as the averaged bulk velocity, which is assumed to be directed along the $x$ axis:
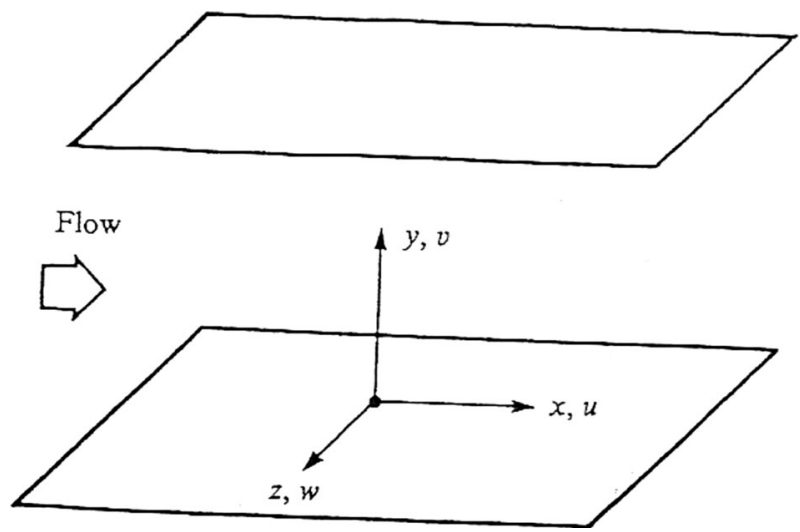

Fig. 1 Representation of the direction of the flow between the walls (up and down), the $\mathrm{x}, \mathrm{y}, \mathrm{z}$-axis (the zero of the $y$-axis is at the middle of the channel), and the $u, v, w$-velocity components of the flow 


$$
U=\frac{1}{V} \int \mathrm{d}^{3} \mathbf{r} v_{x}(\mathbf{r})
$$

Such a choice is particularly convenient in our simulations since the flow is driven by imposing a constant mass flux, which amounts of imposing $\partial_{t} U=0$. Hence, $U$ is constant and its initial value becomes a control parameter that can be used to determine a priori the hydrodynamic Reynolds number $R e$. Equations 2-4 are referred to as the low- $R_{m}$ MHD equations.

\subsection{Numerical Simulations}

The direct numerical simulation method used to solve the low- $R_{m}$ equations is based on the code of Lundbladh et al. (1999). The equations that are solved for the velocity field are the normal velocity equation and the normal vorticity equation similarly to Kim et al. (1987). The other velocity components $u$ and $w$ are obtained from the incompressibility constraint and the definition of the normal vorticity. The pressure is thus eliminated and the number of variables to be solved for is reduced from 4 to 2. The spectral code is based on Fourier representation in the streamwise $(x)$ and spanwise $(z)$ directions, and Chebyshev polynomials in the wall-normal (y) direction. The bulk Reynolds number based on $U$ and the channel half-width, $\delta$, is 2800 , the Reynolds number based on the laminar centerline velocity and $\delta$ is 3300. The Reynolds number, $R e_{\tau}$, based on the wall friction velocity, $u_{\tau}$ and $\delta$ for the hydrodynamic case is approximately 180 similar to the case study by Kim et al. (1987). The mean pressure gradient is adjusted in such a way that a constant mass-flux is maintained throughout a simulation.

One value of the magnetic interaction parameter is used in the streamwise direction of the magnetic field, $N=0.1$, which ensures that the flow will remain turbulent during the calculation. This value of $N$ is selected also similar to the one used by Lee and Choi (2001) for comparison purposes, and the corresponding Hartmann numbers are in the range of $0 \sim 16.6$.

All simulations were performed using $128 \times 128 \times 128$ grid points in a $4 \pi \delta \times 2 \delta \times 4 / 3 \pi \delta$ computational domain similar to the one employed by Moser et al. (1999) for the same $R e_{\tau}$. Dealiasing using the 3/2-rule was employed in the wall-parallel (Fourier) directions. The grid cell sizes, in wall units, depend on the corresponding friction Reynolds number, and thus, on the particular magnetic field used. Table 1 presents a summary of $R e_{\tau}$ and the grid sizes for the simulations. Non-dimensional units marked by the superscript "+" are scaled in wall units and defined as $y^{+}=y u_{\tau} / v$ and $u^{+}=u / u_{\tau}$, respectively, where

$$
u_{\tau}=\sqrt{\left.v \frac{\partial\left\langle u_{x}\right\rangle}{\partial y}\right|_{\text {wall }}}
$$

is the friction velocity. The brackets $\langle\cdot\rangle$ indicate an average in both the wall-parallel directions $x$ and $z$ as well as time. The time is advanced using a combined Crank - Nicolson scheme for

Table 1 Grid resolution and $R e_{\tau}$ for all cases considered

\begin{tabular}{llllllll}
\hline Case & MF direction & $N$ & $H \alpha$ & $R e_{\tau}$ & $\Delta x^{+}$ & $\Delta y_{\min }^{+}$ & $\Delta z_{\max }^{+}$ \\
\hline 1 & - & 0 & 0 & 179.3 & 17.6 & $0.054-4.39$ & 4.39 \\
2 & $x$ & 0.1 & 16.7 & 171.8 & 16.8 & $0.051-4.21$ & 4.21 \\
\hline
\end{tabular}


linear diffusion terms and a four stage Runge - Kutta scheme for the nonlinear terms. As the velocity is advanced at each time step, the corresponding electric potential field is obtained by solving the Helmholtz problem Eq. (4), in spectral space. The Lorentz force in Eq. (2) is added explicitly at every Runge-Kutta substep. The time step is automatically computed using a CFL criterion. Once a statistically steady state is reached, the statistics and time series are averaged both in time and in the homogeneous wall-parallel directions. Based on the streamwise domain size $L_{x}$ and the bulk velocity $U$, Eq. (5), typical integration times are always larger than $1000 L_{x} / U$ meaning that, on average, the fluid crosses more than 1000 times the computational domain.

Periodic boundary conditions are imposed in the wall-parallel directions for all quantities. At the solid walls, located at $y= \pm 1$, no-slip boundary conditions are used for the velocity field. Furthermore, the walls are assumed to be non-conducting. Therefore, the boundary conditions for the current density and the electrical potential are:

$$
\left.j_{y}\right|_{\text {wall }}=-\left.\frac{\partial \phi}{\partial y}\right|_{\text {wall }}=0
$$

where, $j_{y}$ is the wall-normal component of the current density. When there is no externally applied electric field and because of the periodicity of the domain, the total current in the flow domain must be zero.

The hydrodynamic part of the code has been successfully validated and compared against the results of Moser et al. (1999). The MHD part of the code has been first successfully compared against the laminar analytic solution of the Hartmann flow for various $H \alpha$ numbers as well as against the DNS results of Lee and Choi (2001).

The analysis that is performed here is based on the measurement of correlations between pairs of fully turbulent temporal fields, one from the MHD and one from the hydrodynamic case in specific probing locations of the channel. The time series of the three velocity components from both cases were recorded at the locations of the channel found in Table 2, while some representative temporal records are presented in Figs. 2, 3 and 4 for both the hydrodynamic case and the case of the flow under the effect of a streamwise external magnetic field. As it is obvious from the time series, the application of the magnetic field reduces the magnitude of the fluctuations without leading to flow laminarization although the weak turbulence of $\operatorname{Re}_{\tau} \sim 180$. Thus, in principle the magnetic field is acting as the flow to be in a lower Re number.

\section{Methodology}

\subsection{Cross Recurrence Plots (CRP)}

Cross Recurrence Plots was introduced to analyze the dependencies between two different systems or simulations, one with the magnetic field and one without it in the present work. It is an extension of the Recurrence Plot method which was introduced by Eckmann et al. (1987). The Cross Recurrence Plot method compares the two systems states represented by their trajectories $x_{i}$ and $y_{i}$ in a $d$-dimensional 
Table 2 Probing of each i-position on the y-direction of the flow (from the wall up to the centerline of the channel)

\begin{tabular}{llllll}
\hline$i$-point & Position & $i$-point & Position & $i$-point & Position \\
\hline 1 & -0.99812 & 16 & -0.86397 & 31 & -0.48218 \\
2 & -0.99391 & 17 & -0.83822 & 32 & -0.46054 \\
3 & -0.9873 & 18 & -0.82459 & 33 & -0.43862 \\
4 & -0.98311 & 19 & -0.79584 & 34 & -0.39399 \\
5 & -0.97832 & 20 & -0.76517 & 35 & -0.37132 \\
6 & -0.97294 & 21 & -0.74914 & 36 & -0.34842 \\
7 & -0.96698 & 22 & -0.71573 & 37 & -0.30201 \\
8 & -0.96043 & 23 & -0.69838 & 38 & -0.27852 \\
9 & -0.95331 & 24 & -0.66242 & 39 & -0.23106 \\
10 & -0.94561 & 25 & -0.64383 & 40 & -0.20711 \\
11 & -0.93734 & 26 & -0.62486 & 41 & -0.15886 \\
12 & -0.92851 & 27 & -0.5858 & 42 & -0.11022 \\
13 & -0.91911 & 28 & -0.56573 & 43 & -0.0858 \\
14 & -0.90917 & 29 & -0.54532 & 44 & -0.03681 \\
15 & -0.89867 & 30 & -0.52459 & 45 & 0.012272 \\
\hline
\end{tabular}

phase space (Marwan et al. 2007). It is based on phase space reconstruction of both time series embedded in the same phase space. Embedding parameters of both time series are found with the methods of false nearest neighbors (embedding dimension $\mathrm{m}$ ) and average mutual information (time delay $\tau$ ). If $m$ and $t$ of the first time series are not equal with $m$ and $t$ of the second time series then we have to choose the set of parameters with the higher embedding dimension (Marwan et al. 2007). Then, the distances $d_{i j}$ between the reconstructed $\mathbf{x}_{\mathbf{i}}, \mathbf{y}_{\mathbf{j}}$ vectors are calculated using the most known norms, Euclidean $\left(\|\|_{2}\right)$ or Maximum $\left(\|\|_{\infty}\right)$ norms and a matrix is constructed, known as distance matrix $\mathbf{C R}_{i, j}$ (Eq. 8).

$$
\mathbf{C R}_{i, j}(\varepsilon)=\Theta\left(\varepsilon-\left\|\vec{x}_{i}-\vec{y}_{j}\right\|\right), \quad i=1, \ldots N, \quad j=1, \ldots, M
$$

The cross recurrence plot is obtained by setting a cut off distance $\varepsilon$ (threshold) (Marwan and Kurths 2002; Zbilut and Webber 1992). Cross recurrence plots show the duration which both systems trajectories run parallel within a certain threshold $\varepsilon$ in the same phase space. If the trajectories are running parallel (in the trajectory $\vec{y}_{j}$ a state at time $j$ laying near a state on the trajectory $\vec{x}_{i}$ at time $i$ ), then the CRP takes the value of 1 and the corresponding element $(\mathrm{i}, \mathrm{j})$ of the matrix is represented by a black point. On the other hand, if the trajectories are separated by a distance larger than the threshold $\varepsilon, \mathrm{CRP}$ is set equal to zero and the corresponding element of the matrix is represented by a white point (Marwan 2003). Note that $\Theta$ (x) in Eq. (8) is the Heaviside function. The time series are normalized before application of CRP analysis by subtracting the mean value and division by their standard deviation.

So, we focus on sectors of the trajectories which run parallel in a certain threshold during time. This time duration is pictured on a CRP as a diagonal line (black points one after the 

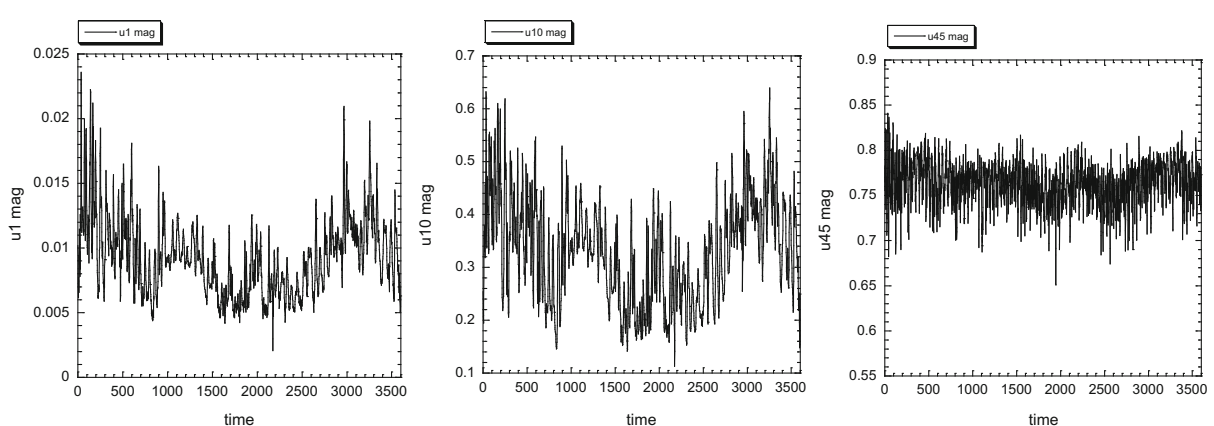

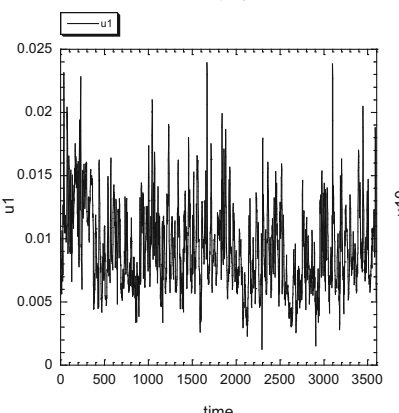

(a)

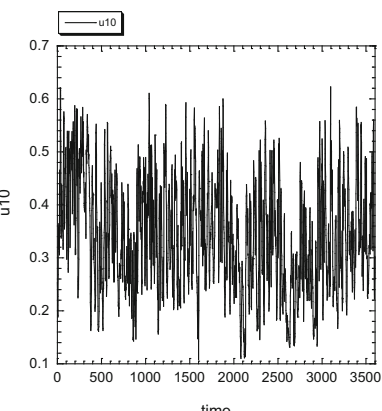

(b)

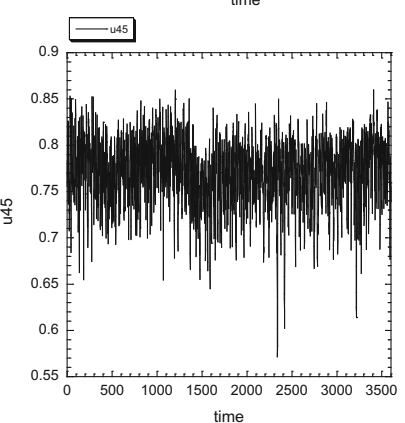

(c)

Fig. 2 Time series with (upper) and without the magnetic field (bottom) of the u-velocity: a near the wall point 1 , positioned at -0.99812 , b further away from the wall point 10 , positioned at -0.94561 , and c point 45 positioned 0.012272 which is placed close to the middle of the channel

other forming a line). The longer the diagonal line, the bigger the time duration the two trajectories run parallel in a certain area of the phase space (threshold). Similarity is interrupted if white points appear, forming white bands (vertical or horizontal) which show that both systems' trajectories, for some time, do not lie parallel in a certain area of the phase space.

\subsection{Cross Recurrence Quantification Analysis (CRQA)}

A very interesting issue is the quantification of the Cross Recurrence Plots (CRPs) for better understanding the non-linear relations between the two time series. Based on quantification of Recurrence Plots (Zbilut and Webber 1992) an extension was introduced by Marwan and Kurths (2002) which measures the parallel lines of a Cross Recurrence Plot. Since a cross recurrence plot does not actually count recurrences between states, but similarities (Marwan and Kurths 2002), the measures we study are the Determinism (DET) and the Average Line Length (L). Determinism (Eq. 9) is the ratio of points forming the diagonal lines to all points of the CRP:

$$
D E T=\frac{\sum_{l=l_{\min }}^{N} l P(l)}{\sum_{l=1}^{N} l P(l)}
$$

Strong similarities are revealed by two states if long diagonal lines are pictured on the CRP, resulting to high values of DET. 

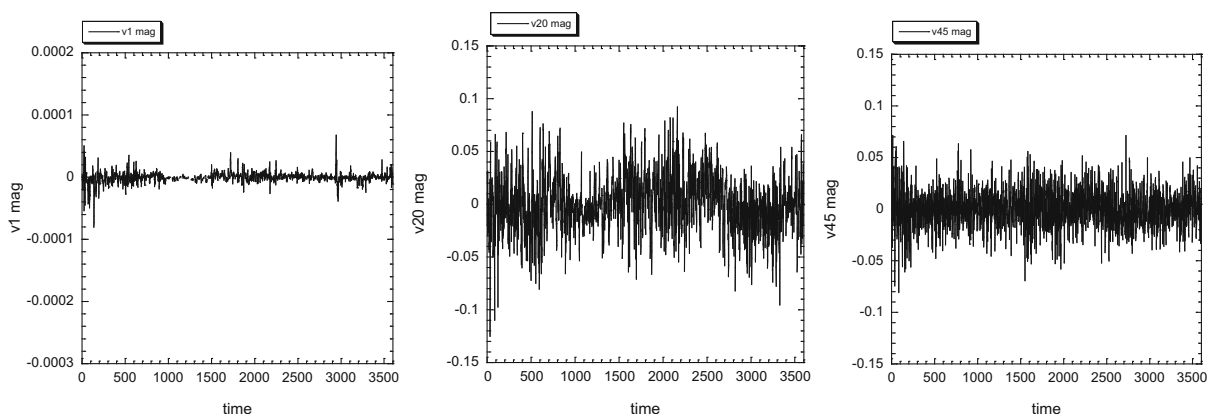

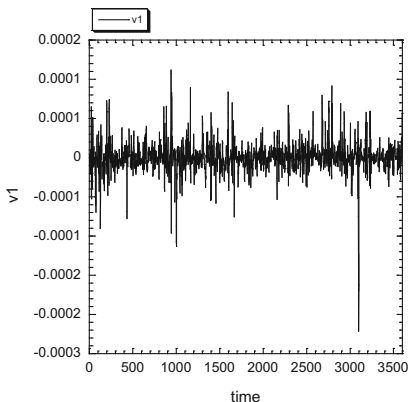

(a)

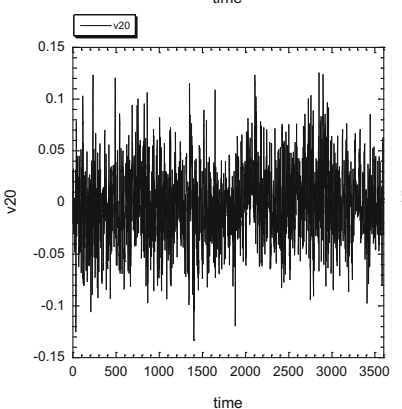

(b)

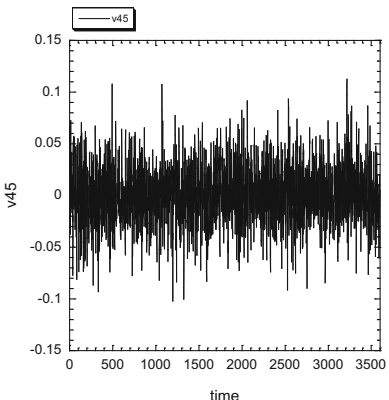

(c)

Fig. 3 Time series with (upper) and without the magnetic field (bottom) of the v-velocity: a near the wall point 1 , positioned at -0.99812 , b further away from the wall point 20 , positioned at $-0.76517, \overline{\text { and } \mathbf{c} \text { close to the }}$ middle of the channel (point 45 located at 0.012272)

Average Line Length (Eq. 10) counts the average length of diagonal lines on the CRP:

$$
L=\frac{\sum_{l=l_{\min }}^{N} l P(l)}{\sum_{l=l_{\min }}^{N} P(l)}
$$

It provides the duration in time. Trajectories of both states close to each other in a certain threshold line with high average length reveal continued similarity during time in both states.

In summary, high values of determinism and average line length depict the trajectories of two states which run close to each other for long time in a certain threshold.

\section{Results}

\subsection{Cross Recurrence Plots}

The method of Cross Recurrence Plots (CRPs) is applied on the three velocity components (Figs. 5, 6 and 7) between time series influenced by the magnetic field (velocities $-\mathrm{m}$ ) and without the magnetic field (velocities - wm), that were recorded at the same probing locations of the channel (Table 2). Initially, the embedding parameters of all time series by applying average mutual information and false nearest neighbor methods (Kennel et al. 1992; Kantz and 


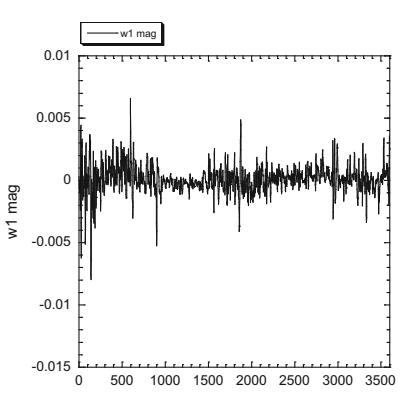

time

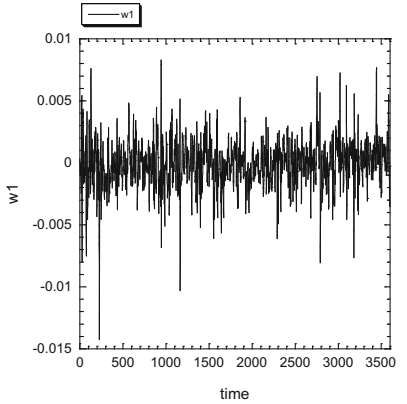

(a)

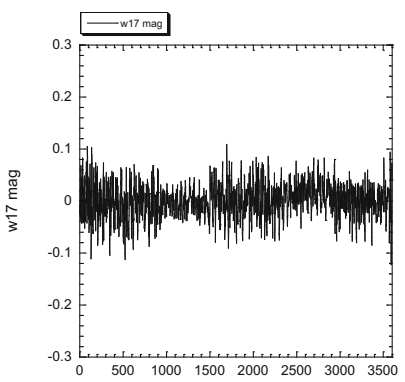

time

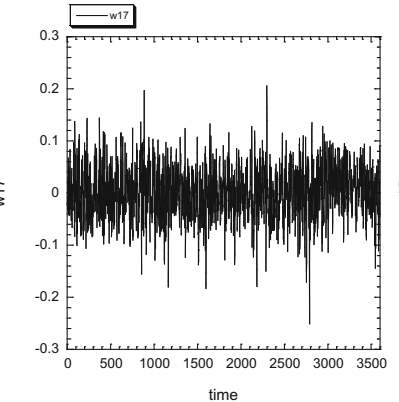

(b)

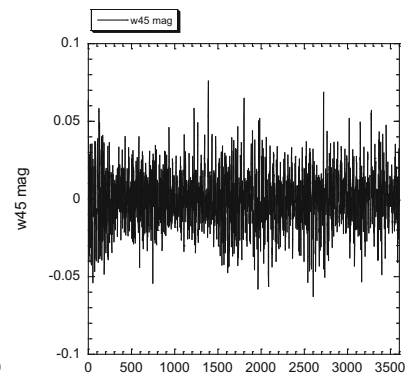

time

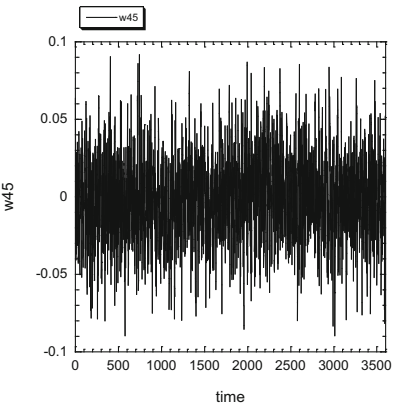

(c)

Fig. 4 Time series with (upper) and without the magnetic field (bottom) of the w-velocity: a near the wall point 1 , positioned at -0.99812 , $\mathbf{b}$ further away from the wall point 17 , positioned at -0.83822 , and $\mathbf{c}$ close to the middle of the channel (point 45 positioned at 0.012272 )

Schreiber 2004) were found on the same probing positions for the three velocity components, then the CRPs were constructed from the trajectories lying in the same phase space. The measurements were performed with the Cross Recurrence Plot Toolbox for Matlab, ver. 5.12, release 25 (2014). The embedding dimension $m$ was set to 15 and time lags varied for the $\mathrm{x}$ direction $7 \leq \tau \leq 20$, for the y direction $3 \leq \tau \leq 10$ and for the $\mathrm{z}$-direction $3 \leq \tau \leq 9$. In a next step, by quantifying the information of those plots using the method of Cross Recurrence Quantification Analysis (CRQA), we analyze the parallel structures (diagonals) of CRPs (trajectories of the phase space which run parallel for some time). We must draw the reader's attention that only CRPs representative of the characteristic behavior observed in each spatial region are presented in Figs. 5, 6 and 7.

First, we study the behavior of the u-velocity component (Fig. 5) where many lines parallel to the main diagonal are observed (Fig. 5a) at probing positions almost near the wall (u1-u9; positions:- 0.99812 to -0.95331 ). The large diagonal lines (for example in the region 1000 1500) indicate that close to the walls the system states at the same channel y position with magnetic and without magnetic field are closely related for quite long times, although it seems that there are regions where such a relation is valid for shorter times since the diagonals are shorter (see for example the regions $0-1000$ and 2500-3000). These long lines lead to large values of DET as we are going to see in the next section. This behavior of CRPs indicates the long lasting memory of the events near the wall as a result of small velocities in the viscous sublayer and as the probe enters in the buffer layer. Moreover, such a behavior can be related to the weak fluctuations of the case with the magnetic field 


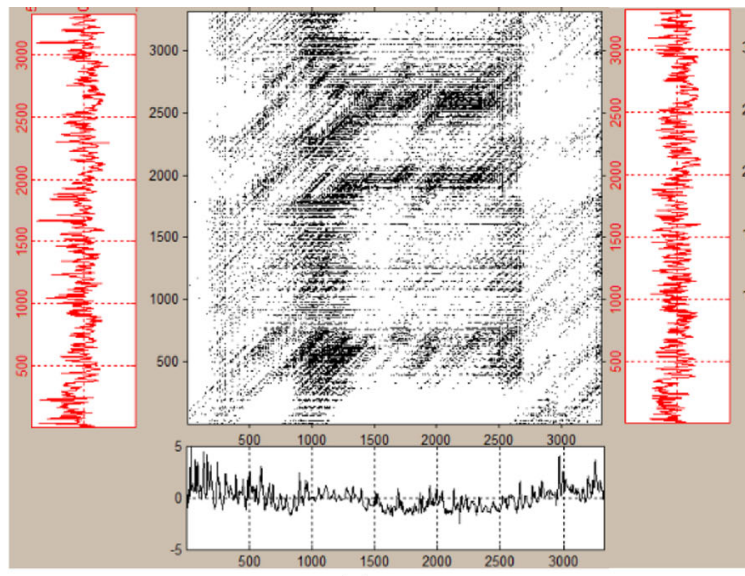

(a)

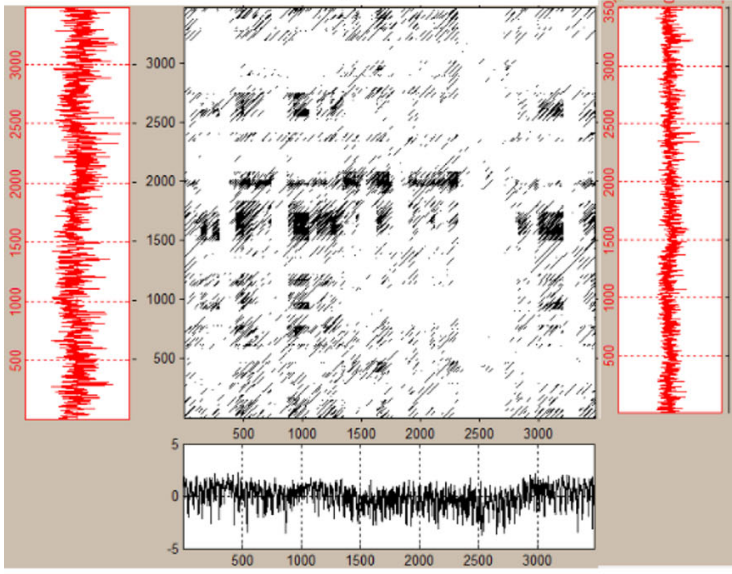

(c)

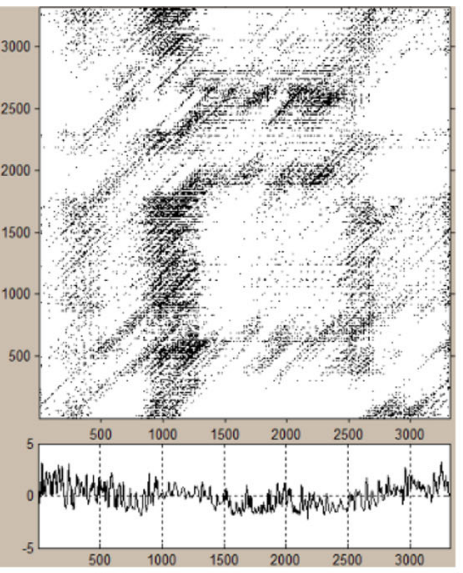

(b)

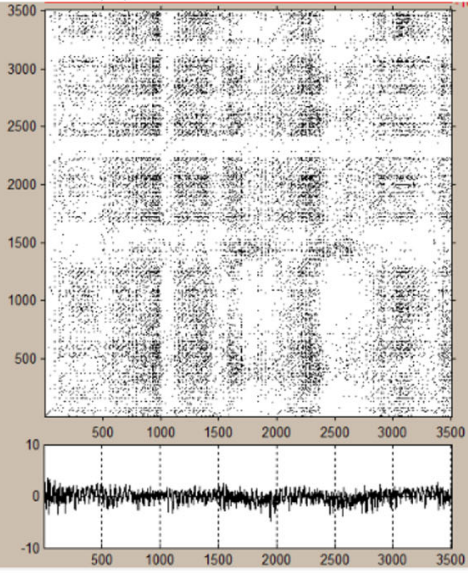

(d)

Fig. 5 CRPs between time series without (shown at the small plot at the left along the y-axis of each plot) and with the magnetic field (shown at the small plot at the bottom along the x-axis of each plot) for u-velocity component, a near the wall point $\mathrm{u} 1$ (position -0.99812), b further away from the wall point $\mathrm{u} 10$ (position -0.94561 ), c point u32 (position -0.48218 ) located at the critical area around -0.4 which is common in all directions, and d point $\mathrm{u} 45$ (position 0.012272 ) placed close to the middle of the channel

As we move to regions further away from the wall (u10-u22; positions -0.94561 to -0.71573 ), we can see that the length of the diagonals gets gradually smaller and white regions make their appearance (Fig. 5b), indicating that the corresponding states (with and without magnetic field) are related for shorter times. Approximately, in the middle of the region studied (1/4 channel) and up to the channel centerline (u23-u45; position: -0.69838 to 0.012272) CRPs contain small lines. In fact this behavior corresponds to the laminarization event due to the magnetic field, found also close to the wall in Fig. 5a between $\mathrm{t}=1000-1500$. A modification of CRPs' texture is observed at probing locations $\mathrm{u} 31-\mathrm{u} 37$ (position: -0.48218 to -0.30201 ) where there are more parallel lines and close to each other (more dense patterns are observed). Approaching the middle of the channel (u35-u45; position: -0.37132 to 0.012227) CRPs contain more isolated points than diagonal lines (which become very small), 


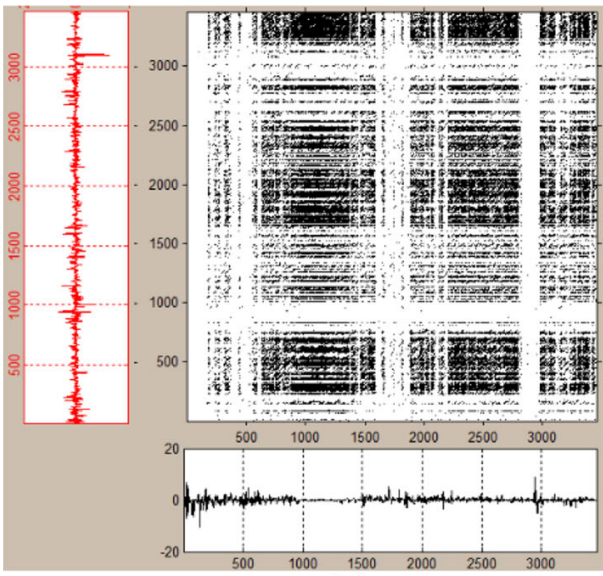

(a)

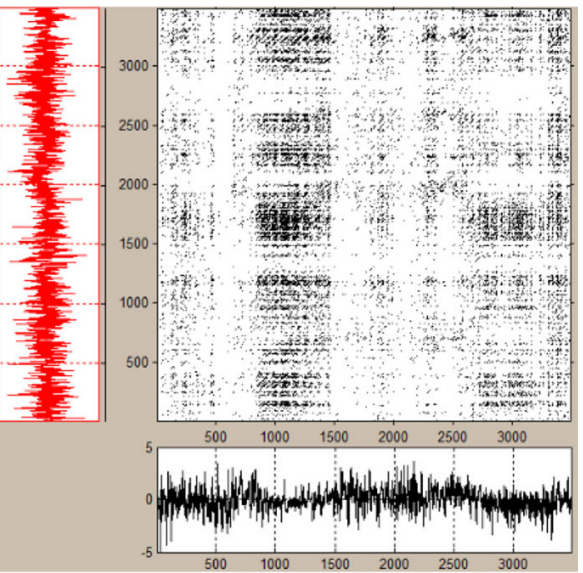

(b)

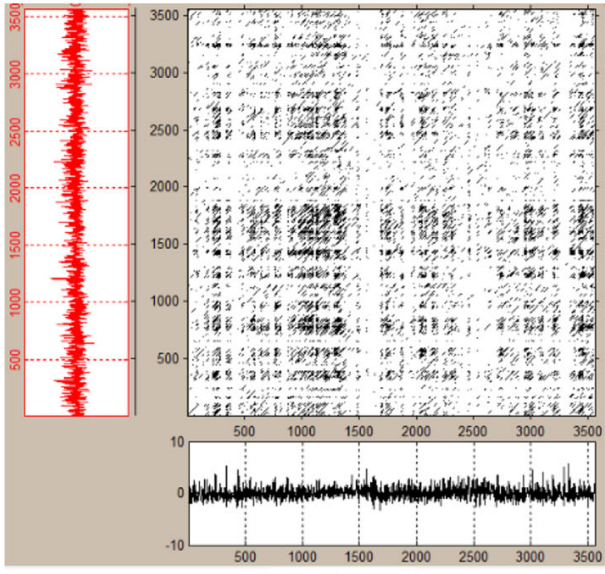

(c)
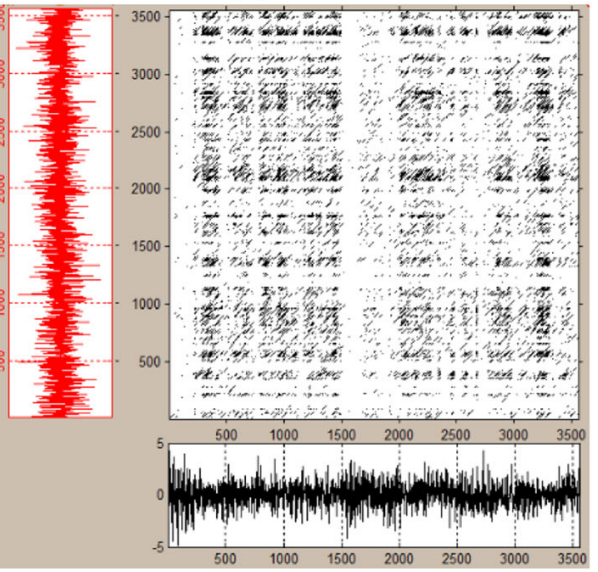

(d)

Fig. 6 CRPs between time series without (shown at the small plot at the left along the y-axis of each plot) and with the magnetic field (shown at the small plot at the bottom along the x-axis of each plot) for the v-velocity component, a near the wall point v1 (position -0.99812), b further away from the wall point v20, (position -0.76517 ), c point $\mathrm{v} 32$ (position -0.48218 ) of the critical area around -0.4 which is common in all directions, and $\mathbf{d}$ point $\mathrm{v} 45$ (position 0.012272 ) located close to the middle of the channel

with an exception of two plots (u37, u38; position: -0.30201 and -0.27852$)$ where a number of parallel lines make their appearance (Fig. 5d). In the middle of the channel, we can see that the lengths of the diagonals are even smaller indicating less related states (for very short times). This overall behavior shows that the flow is more affected in the middle of the channel by the presence of the magnetic field, and thus, the corresponding states (with and without magnetic field) are less related.

In fact, these results are in agreement with the results of Lee and Choi (2001) who observed that close to the walls all the velocity curves collapse to the wall-law of the hydrodynamic viscous sublayer. This means that very close to the wall no significant deviation from the usual hydrodynamic behavior of the turbulent channel flow is observed. For the hydrodynamic case and the cases of the weak magnetic field the mean velocities are similar. However, away from 


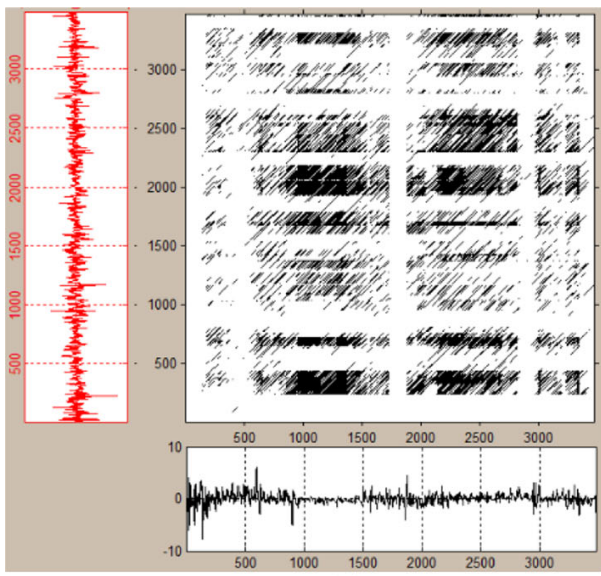

(a)

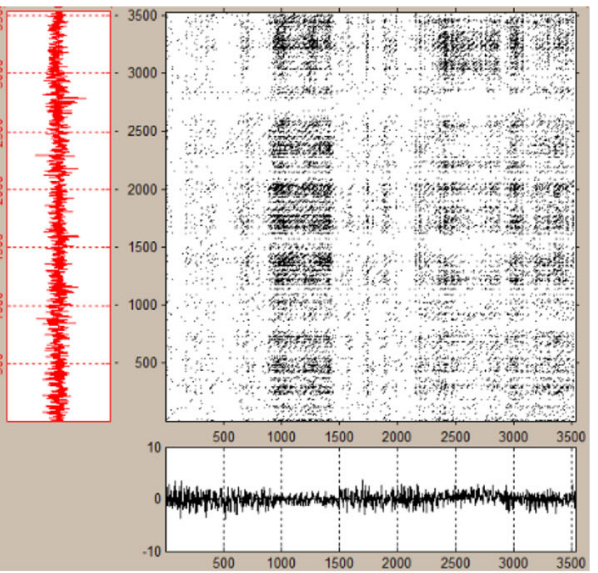

(b)

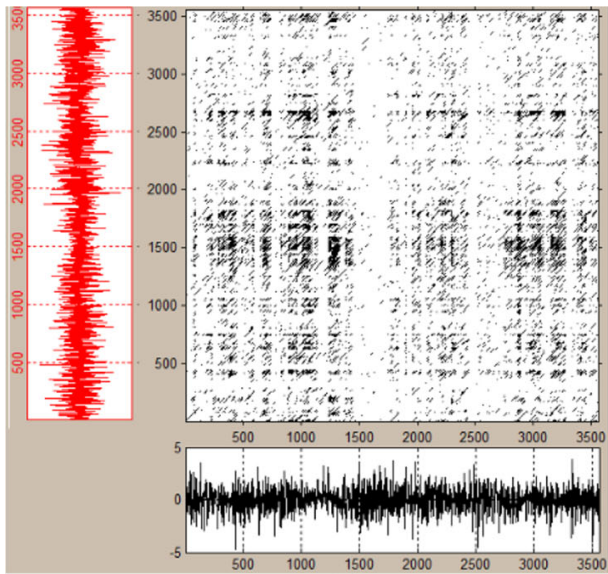

(c)

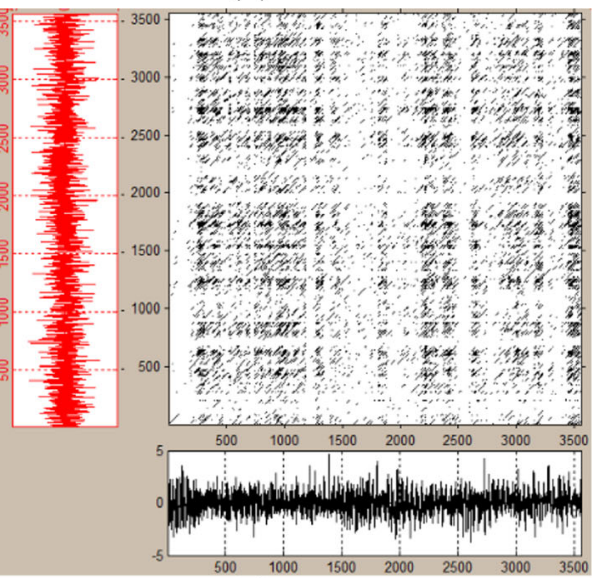

(d)

Fig. 7 CRPs between time series without (shown at the small plot at the left along the y-axis of each plot) and with the magnetic field (shown at the small plot at the bottom along the x-axis of each plot) for the w-velocity component, a near the wall point w1 (positioned -0.99812), b further away from the wall point w17 (position -0.83822 ), c point w32 (position -0.48218 ) at the critical area around -0.4 which is common in all directions, and $\mathbf{d}$ point w45 (position 0.012272) located close to the middle of the channel

the walls a flattening and deviation of the usual logarithmic law (i.e., $<\mathrm{u}^{+}>=2.5 \ln \mathrm{y}^{+}+5.5$ ) is observed with the increase of the magnetic field.

For the v-velocity component (Fig. 6a) near the wall probing locations the length of diagonal lines is relatively small compared to the same locations concerning the u component (Fig. 5a) and a little further (v1-v19; positions: -0.99812 to -0.79584 ), but with small length. This means that the states in the v-velocity are less related than the corresponding ones for the $\mathrm{u}$ component. We believe that again this indicates the laminarization due to magnetic breaking that is observed in the detailed analysis of such simulations. In the middle of the simulation region and up to the middle of the channel (v20-v45; positions:-0.76517 to 0.01272) the lines parallel to the main diagonal become fewer and shorter, and the corresponding dense patterns they form are vanishing, and isolated points appear. In this area, we can separate v31 to v33 
(positions: -0.48218 to -0.43862 ) where CRPs contain longer lines than the other plots in the same region. This behavior reflects the existence of a large number of statistically independent events due to the fully developed turbulence in the buffer layer and the log-law region of the flow. As it is obvious in all the CRPs of the v-velocity, their patterns are darker than of the uvelocity ones. This increased cross correlation is due to the direct weakening of the velocity fluctuations due to the magnetic field in the wall-normal direction. The same effect due to the magnetic field is also discussed for the spanwise velocity fluctuations of Fig. 7 in the next paragraph.

Looking at the CRPs of the w-velocity component (Fig. 7a) which concern the probing locations near the wall and a little further (w1 - w16; position: -0.99812 to -0.86397 ) we observe many small lines parallel to the main diagonal, so dense to each other that form black patterns. However, they are shorter than the ones obtained in the u component (Fig. 5a). Those patterns seem to vanish gradually as we move further away from the wall (w17-w45; positions: -0.83822 to 0.012272 ) and CRPs (Fig. 7b) contain shorter diagonals and isolated points as we approach the middle of the channel (u45). However, there are CRPs at points (w22-w25; positions: -0.71573 to -0.64383 ), (w32-w35; positions: -0.46054 to -0.37132 ) on which we can distinguish small lines parallel to the main diagonal. As the streamwise magnetic field acts only in the turbulent fluctuations of the w-velocity by reducing their magnitude, the effect is more obvious close to the wall, where the velocity fluctuations are injected into the flow. Moreover, the turbulent events at the other distances off the wall are much less affected.

So, the most similar states (velocity before and after the influence of the magnetic field) seem to be near the walls, inside the viscous sublayer, in all directions, since the plots with the most parallel diagonal lines are positioned to those points. In these areas, the magnetic field is less affecting the flow as both the mean velocity and its fluctuations are small. States begin to lose their similarity to the probing locations further away from the wall and approaching the center of the flow, with an exception of the region around position $y=-0.4$ (Fig. 5c, 6c, 7c) or at $\mathrm{y}^{+} \sim 100$ where the mean velocity develops a plateau in the central part of the channel.

\subsection{Cross Recurrence Quantification Analysis (CRQA)}

The visual analysis of CRPs by a careful observation is interesting and provides, as we have already seen, an interesting insight on the physical behavior of the two systems (with and without the magnetic field) as well as its spatial variation across the channel. However, CRPA which quantifies the information on the plots can provide an additional insight on the underlying physical phenomena. Thus, we calculated two measures of similarity related to the lines parallel to the main diagonal: Determinism and Average Line length (Fig. 8). Their values have been extracted, considering Recurrence Rate valued from 1 to $1.3 \%$ (Webber and Zbilut 1994).

The results for Determinism and Average line length are plotted as a function of the probing position in Fig. 8 where three regions can be distinguished. One corresponds to the region almost near the wall, the region where the probing positions are further away from the wall and the region where the probing positions are located in the central area of the channel. The results are plotted for each direction (x,y, z). More specifically, from the results of CRQA for the u-velocity (Fig. 8a) it can be seen that at points almost near the wall (u1-u9; position: -0.99812 to -0.95331$)$ values of Determinism are high (DET $=0.85-0.91)$ and the same occurs for the Average Line Length which is up to 3.6 ( $L=3.27$ to 3.6), in accordance with the qualitative observations on Fig. 5a. At probing locations further away from the wall (u10-u22; 


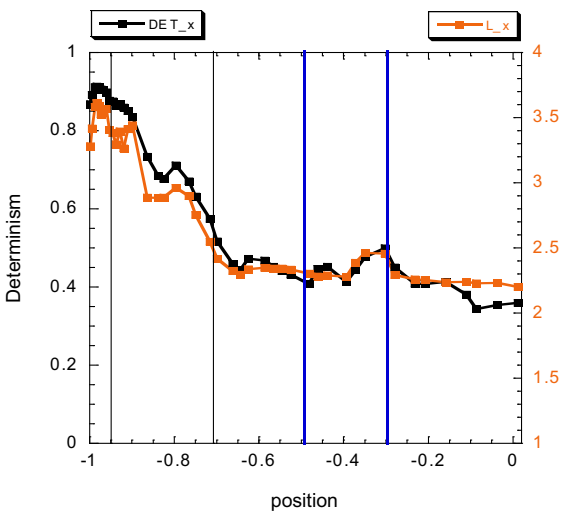

(a)

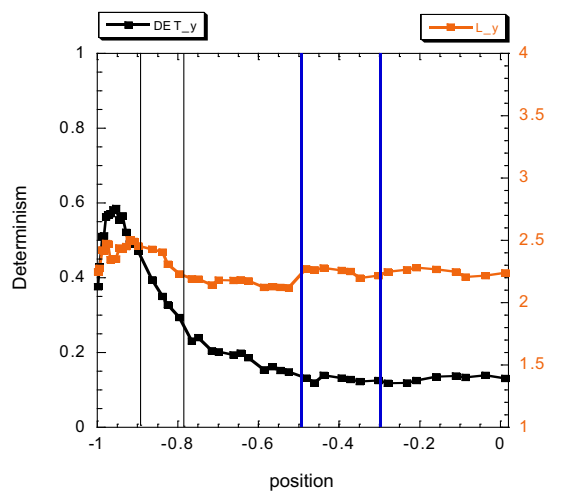

(b)

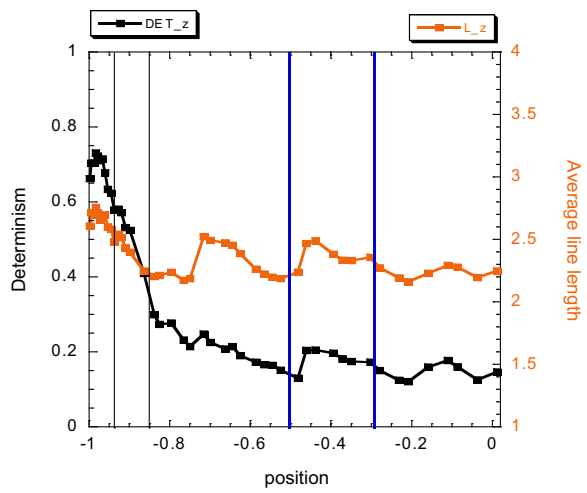

(c)

Fig. 8 Cross Recurrence Quantification Analysis between time series with and without magnetic field, determinism - DET and Average Line Length - L for: a u-velocity, b v-velocity, and $\mathbf{c}$ w-velocity

position: -0.94561 to -0.71573 ) values of determinism and average line length fall to 0.57 and 2.54 , respectively $(\mathrm{DET}=0.57-0.84, L=2.55-3.42)$ as turbulence is stronger and the two time series have only fewer and shorter in time correlations. At probing locations placed at the central part of the channel ( $\mathrm{u} 23-\mathrm{u} 45$; position: -0.69838 to 0.012272 ) determinism and average line length take the lowest values of all u-velocity time series (DET $=0.34-0.5, L=$ 2.2-2.4); in this region the time series are determined by shorter memory events of the mean turbulent streamwise velocity. It is worth saying that there is a critical area (u31-u37; position: -0.48218 to -0.30201 ) where different behavior is observed compared to the behavior of this examined region. At this area, a mixed behavior is found close to $\mathrm{y}^{+} \sim 28$ at the interface between the buffer layer and the log-law region.

In the case of CRQA results for the v-component (Fig. 8b), we can again distinguish three regions. In the region of probing locations almost near the wall (v1-v15; position: -0.99812 to $-0.89867)$ Determinism attains values up to $0.58(\mathrm{DET}=0.4-0.58)$ and the Average Line Length values up to 3.6 ( $L=3.27$ to 3.6). The region of probing locations further away from the wall region (v16-v19; position: -0.86397 to -0.79584 ) where values of determinism and average line length decrease to values close to 0.3 and 2.21 , respectively (DET $=0.3-0.4, L=$ 
2.21-2.43). For probing locations in the central region of the channel (v20-v45; position: -0.76517 to 0.012272 ) determinism and average line length present lower values than at points near the wall region ( $\mathrm{DET}=0.11-0.23, L=2.18-2.28$ ). It is worth saying again that there is a critical area (v31-v37; position: -0.48218 to -0.30201 ) the same as in u-component where again different behavior is observed compared to the behavior of this examined region (peak).

Three regions can also be observed for the w-velocity (Fig. 8c). The region of probing locations almost near the wall (w1 - w10; position:-0.99812 to -0.94561) where Determinism attains values up to $0.73(\mathrm{DET}=0.57-0.73)$ and Average Line Length values up to 2.77 ( $L=2.4$ to 2.77). In the second region where probing locations are further away from the wall (w11-w16; position: -0.93734 to -0.86397 ) values of determinism and average line length fall to 0.4 and 2.2 , respectively ( $\mathrm{DET}=0.4-0.58, L=2.2-2.54$ ). In the extensive central part of the channel (w17-w45; position: -0.83822 to 0.012272 ) determinism gives lower values than at probing locations near the wall region $(\mathrm{DET}=0.11-0.23)$ in contrast to average line length which values are almost the same to those further from the wall $(L=2.19-2.5)$. For probing locations in the central region of the channel (w22-w45; position -0.71573 to 0.012272 ) three critical areas are observed, (w22 - w24; position: -0.71573 to -0.66242 ), the common separated with blue lines (v31-v37; position: -0.48218 to -0.30201$)$ and (w38-w45; position: -0.27852 to 0.012272 ) along this region (three picks). Moreover, it is observed that both the Determinism and average line length have similar variation along the channel for both the $\mathrm{v}$ and w-velocity components which we attribute to a similar reduction of their fluctuations by the magnetic field.

In Fig. 9, we summarize the results for the three velocity components for comparison reasons. It is found that $\mathrm{u}$-velocity has the highest values of DET (Fig. 9a) among the three components all along the probing locations, while $\mathrm{v}$ and $\mathrm{w}$-velocities are found to have a quite similar behavior, with the exception of the region near the wall. In this near the wall region the w-velocity DET values are higher than those of the v-velocity since the weak fluctuations of both w-velocity time series are more correlated near the wall. This correlation is reversed close to the wall for the v-velocity because of the random production of streak structures independent of the action of the magnetic field.

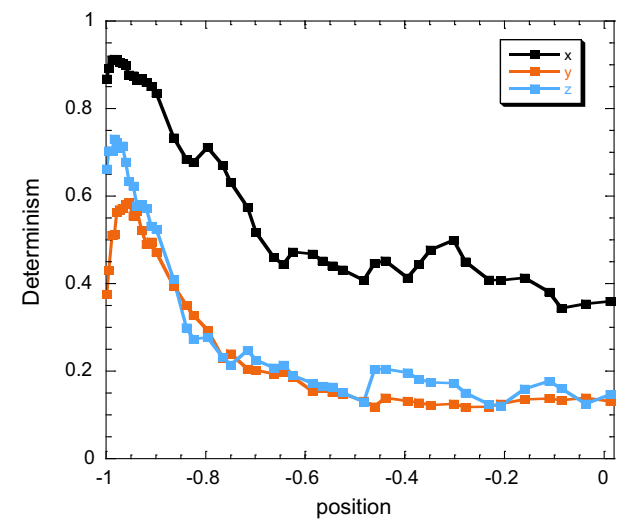

(a)

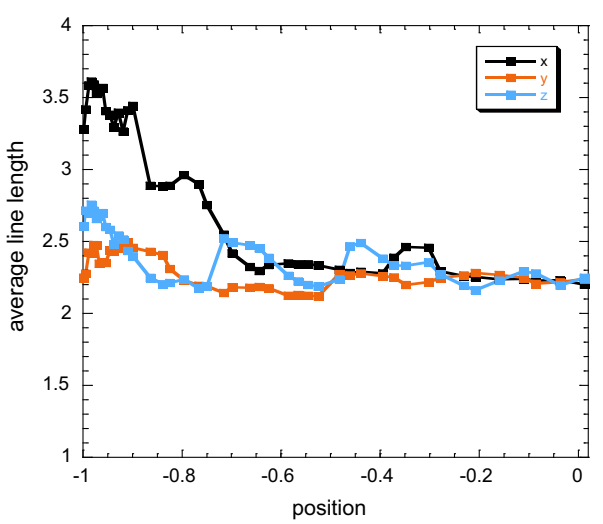

(b)

Fig. 9 Cross Recurrence Quantification Analysis between time series with and without magnetic field along the u, v, w-velocity components: a determinism - DET, b Average Line Length - L 
As far as the Average Line Length is concerned (Fig. 9b), we can observe higher values for the u-velocity compared to the other velocity components only close to the walls $(y>0.75)$. In the central part of the channel, their values are quite similar for all three directions although some minor differences are observed. These results indicate that in the region close to the wall both velocity time series are strongly related and present similarities for long times due to their weak fluctuations.

\section{Conclusions}

Cross Recurrence plots can reveal similarities/correlations between the two states (with magnetic field and without magnetic field) in a visual way. The states of the u-velocity seem to present the higher similarity in phase space since CRPs contain more diagonal lines parallel to the main diagonal and this is true especially for the region close to the wall. Similarity seems to be gradually lost when we go further away from the walls and approach the middle of the channel where states are more different than similar (more isolated points on CRPs than small diagonal lines).

CRPs of $\mathrm{v}$ and $\mathrm{w}$-velocity components present also a significant number of lines parallel to the main diagonal for points near the wall, however, they are shorter than those corresponding to u-velocity. An interesting issue is that these diagonal parallel lines are relatively small and very dense forming black patterns, meaning that the values of one of the two states are close to each other for very small times indicating the fact that they found themselves in similar states for small durations compared to the u-velocity where the flow in driven.

The quantification analysis of the above CRPs as represented by the two quantities Determinism and Average Line Length, which have to do with the amount and the length of diagonal lines parallel to the main diagonal, gave us a more detailed picture about the spatial variation of state similarities/correlations. The long diagonal lines of the CRPs parallel to the main diagonal of the $u$-velocity result in high values of determinism and their average length is large enough to say that near the walls the two states present similar states that evolve in a similar way (not in the same real time however) than as we go further away from the wall and towards the channel centerline where this correlation reduces. This difference as a function of the distance reflects the underlying dynamics of the system, where close to the wall the weak magnetic field is less affecting the also weak flow. In contrast, in the central part of the channel the effect of the magnetic field is stronger because of its increased breaking due to the higher velocities.

The same picture about the state correlations and Determinism and Average line Length is observed also for the other two velocity components at least qualitatively. The values are lower than that of the u-velocity, but relatively high ones are observed near the wall (both for $\mathrm{v}$ and w-velocities) revealing similarity/correlation between states. Going further away from the wall and approaching the channel centerline values of determinism and average line length, they decrease due to the stronger influence from the magnetic field as turbulence is more important in these regions.

Along all velocity components at the region located between the middle of the simulation region (1/4 channel) up to the channel centerline some critical areas are observed with maximum values of determinism and average line length. The most important lie at the area (u31, v31, w31 - u37, v37, w37; position:-0.48218 to -0.30201) which is common in all components. Observing CRPs of those positions are different compared to their neighbor 
CRPs (more parallel lines than their neighbor CRPs) mean that at this area the two states are more similar than they could be at their neighbor positions.

Summarizing the method of Cross Recurrence Plots and Cross Recurrence Quantification Analysis can reveal not only nonlinear similarities between two different states, but can also reveal the spatial variation of these correlations.

Acknowledgments I.E.S. work is part of the EuroFusion research activities supported by the European Commission in the framework of the Euratom-Hellenic Republic Association. The DNS code was provided by Dr. P. Schlatter whose help is greatly acknowledged. An initial version of the paper has been presented in the 12th International Conference on Protection and Restoration of the Environment, Skiathos Island, Greece, June 29 to July 3, 2014.

\section{References}

Addoa PM, Billio M, Guégan D (2013) Nonlinear dynamics and recurrence plots for detecting financial crisis. N Am J Econ Finance 26:416-435

Boeck T, Krasnov D, Zienicke E (2007) Numerical study of turbulent magnetohydrodynamic channel flow. J Fluid Mech 572:179-188

Cazares-Ibanez E, Vazquez-Coutino GA, Garcia-Ochoa E (2005) Application of recurrence plots as a new tool in the analysis of electrochemical oscillations of copper. J Electroanal Chem 583(1):17-33

Cross Recurrence Plot Toolbox for Matlab (2014), ver. 5.12, release 25 http://www.agnld.uni-potsdam.de/ $\sim$ marwan/toolbox/. Accessed Jan 2014

Crowley P and Aaron S (2010) A new approach to analyzing convergence and synchronicity in growth and business cycles: cross recurrence plots and quantification analysis. Munich Personal RePEc Archive, MPRA Paper No. 23728

Deng LH, Li B, Zheng YF, Cheng XM (2013) Relative phase analyses of $10.7 \mathrm{~cm}$ solar radio flux with sunspot numbers. New Astronomy, 23-24:1-5

Eckmann J-P, Kamphorst SO, Ruelle D (1987) Recurrence plots of dynamical systems. Europhys Lett 4(9):973977

Fabretti A, Ausloos M (2005) Recurrence plot and recurrence quantification analysis techniques for detecting a critical regime. Examples from financial market indices. Int J Mod Phys C 16(5):671-706

Ganapathy R, Rangarajan G, Sood AK (2007) Granger causality and cross recurrence plots in rheochaos. Phys Rev E 75:016211

Giuliani A, Benigni R, Zbilut JP, Webber CL Jr, Sirabella P, Colosimo A (2002) Nonlinear signal analysis methods in the elucidation of protein sequence-structure relationships. Chem Rev 102(5):1471-92

Hartmann J (1937) Theory of the laminar flow of an electrically conductive liquid in a homogeneous magnetic field. Matematisk-fysiske Medd 15(6):1-28

Hartmann J, Lazarus F (1937) Experimental investigations on the flow of mercury in a homogeneous magnetic field. Matematisk-fysiske Medd 15(7):1-45

Kantz H, Schreiber T (2004) Nonlinear Time Series Analysis, Cambridge University Press, second edition

Karakasidis TE, Fragkou A, Liakopoulos A (2007) System dynamics revealed by recurrence quantification analysis: application to molecular dynamics simulations. Phys Rev E 76(2):021120

Kennel MB, Brown R, Abarbanel HDI (1992) Determining embedding dimension for phase-space reconstruction using a geometrical construction. Phys Rev A 45(6):3403-3411

Kim J, Moin P, Moser R (1987) Turbulent statistics in fully developed channel flow at low Reynolds number. J Fluid Mech 177:133-166

Lee D, Choi H (2001) Magnetohydrodynamic turbulent flow in a channel at low magnetic Reynolds number. J Fluid Mech 439:367-394

Li K, Li ZM (2013) Cross recurrence quantification analysis of precision grip following peripheral median nerve block. Journal of neuroengineering and rehabilitation, 10(March 2013): Article number 28

Lundbladh A, Berlin S, Skote M, Hildings C, Choi J, Kim J, Henningson DS (1999) An efficient spectral method for simulation of incompressible flow over a flat plate. Tech. Rep. TRITA-MEK 1999:11, KTH Stockholm, Sweden

Marwan N (2003) Ph.D. Thesis, University of Potsdam 
Marwan N, Kurths J (2002) Nonlinear analysis of bivariate data with cross recurrence plots. Phys Lett A 302(56):299-307

Marwan N, Meinke A (2004) Extended recurrence plot analysis and its application to ERP data. Int J Bifurcation Chaos 14(2):761-771

Marwan N, Thiel M, Nowaczyk NR (2002a) Cross recurrence plot based synchronization of time series. Nonlinear Process Geophys 9(3-4):325-331

Marwan N, Wessel N, Meyerfeldt U, Schirdewan A, Kurths J (2002b) Recurrence-plot-based measures of complexity and their application to heart-rate-variability data. Phys Rev E 66(2):026702

Marwan N, Tauth MH, Vuille M, Kurths J (2003) Comparing modern and Pleistocene ENSO-like influences in NW Argentina using nonlinear times series analysis methods. Clim Dyn 21(3-4):317-326

Marwan N, Romano MC, Thiel M, Kurths J (2007) Recurrence plots for the analysis of complex systems. Phys Rep 438(5-6):237-329

Moreau R (1998) Magnetohydrodynamics. Kluwer Academic Publishers

Moser RD, Kim J, Mansour NN (1999) Direct numerical simulation of turbulent channel flow up to Re $\tau=590$. Phys Fluids 11(4):943-945

Nichols JM, Trickey ST, Seaver M (2006) Damage detection using multivariate recurrence quantification analysis. Mech Syst Signal Process 20(2):421-437

Ponyavin DI and Zolotova NV (2004) Cross Recurrence Plots Analysis of the North-South Sunspot Activities. Proceedings of the International Astronomical Union 2004(IAUS223):141-142

Riley MA, Balasubramaniam R, Turvey MT (1999) Recurrence quantification analysis of postural fluctuations. Gait Posture 9(1):65-78

Sarris IE, Zikos GK, Grecos AP, Vlachos NS (2006) On the validity of the low magnetic Reynolds number approximation in MHD natural convection heat transfer. Numer Heat Transfer: Part B - Fundam 50(2):157180

Sarris IE, Kassinos SC, Carati D (2007) Large-eddie simulations of the turbulent Hartmann flows close to the transitional regime. Phys Fluids 19:085109

Satake S, Kunugi T, Takase K, Ose Y (2006) Direct numerical simulation of turbulent channel flow under a uniform magnetic field for large-scale structures at high Reynolds number. Phys Fluids 19:125106

Serrà J, Serra X, Andrzejak RG (2009) Cross recurrence quantification for cover song identification. New J Phys 11:093017

Shockley K, Butwill M, Zbilut JP, Webber CL Jr (2002) Cross recurrence quantification of coupled oscillators. Phys Lett A 305(1-2):59-69

Sparavigna A (2008) Recurrence plots of sunspots, solar flux and irradiance. http://arxiv.org/ftp/arxiv/papers/ 0804/0804.1941.pdf

Strozzi F, Zaldiovar J-M, Zbilut JP (2002) Application of nonlinear time series analysis techniques to highfrequency currency exchange data. Phys A 312(3-4):520-538

Wang C, Zhong Z, Jiaqiang E (2012) Flow regime recognition in spouted bed based on recurrence plot method. Powder Technol 219:20-28

Webber CL Jr, Zbilut JP (1994) Dynamical assessment of physiological systems and states using recurrence plot strategies. J Appl Physiol 76(2):965-973

Zbilut J, Webber C Jr (1992) Embeddings and delays as derived from quantification of recurrence plots. Phys Lett A 171(3-4):199-203

Zbilut JP, Giuliani A, Colosimo A, Mitchell JC, Colafranceschi M, Marwan N, Webber CL Jr, Uversky VN (2004) Charge and hydrophobicity patterning along the sequence predicts the folding mechanism and aggregation of proteins: a computational approach. J Proteome Res 3(6):1243-1253 